\title{
Analytical Redundancy Based Predictive Fault Tolerant Control of a Steer-By-Wire System Using Nonlinear Observer
}

\author{
Sohel Anwar ${ }^{+}$and Wei Niu ${ }^{++}$ \\ ${ }^{+}$Purdue School of Engineering and Technology \\ ${ }^{++}$Lugar Center for Renewable Energy \\ Indiana University - Purdue University Indianapolis
}

\begin{abstract}
A nonlinear observer based analytical redundancy methodology is presented for fault tolerant control of a steer by wire (SBW) system. A long-range predictor based on Diophantine identity has been utilized to improve the fault detection efficiency. The overall predictive fault tolerant control strategy was then implemented and validated on a steer by wire hardware in loop bench. The experimental results showed that the overall robustness of the SBW system was not sacrificed through the usage of analytical redundancy for sensors along with the designed fault detection algorithm. Moreover, the experimental results indicate that the faults could be detected faster using the developed analytical redundancy based algorithms for attenuating-type faults.
\end{abstract}

